\title{
Gastrite hemorrágica por alergia ao leite de vaca: relato de dois casos
}

\author{
Hemorrhagic gastritis due to cow's milk allergy: report of two cases \\ Rodrigo Strehl Machado ${ }^{1}$, Elisabete Kawakami ${ }^{2}$, Soraya Goshima ${ }^{1}$, \\ Francy Reis Patrício ${ }^{3}$, Ulysses Fagundes Neto ${ }^{4}$
}

\section{Resumo}

Objetivo: descrever dois lactentes com gastrite hemorrágica, devido à alergia ao leite de vaca.

Descrição dos casos: ambos os pacientes apresentavam hematêmese, vômitos e desnutrição. Os pacientes apresentavam infiltrado eosinofílico em mucosa gástrica biopsiada e resolução dos sintomas após o início de dieta com exclusão da proteína do leite de vaca.

Comentários: gastrite hemorrágica devido à alergia ao leite de vaca é um diagnóstico incomum. Até o momento foram relatados 10 pacientes com gastrite alérgica, e as principais manifestações clínicas são vômitos, desnutrição, anemia e hematêmese. A gastrite por alergia ao leite de vaca pode apresentar-se em diferentes graus de gravidade, e pode ser subdiagnosticada em muitos pacientes. A menos que o tratamento correto seja iniciado, o paciente desenvolverá desnutrição protéico-calórica grave e anemia. Doença alérgica do trato gastrointestinal superior deve ser considerada em todos os lactentes com vômitos recorrentes, particularmente se complicado com hematêmese. O diagnóstico de gastrite alérgica é clínico, auxiliado pela endoscopia e anatomia patológica.

J Pediatr (Rio J) 2003;79(4):363-8: gastrite hemorrágica, alergia ao leite de vaca, lactentes.
1. Médico Pediatra, Mestre em Medicina pela Universidade Federal de São Paulo, Escola Paulista de Medicina.

2. Professora de Gastroenterologia Pediátrica, Departamento de Pediatria, Universidade Federal de São Paulo, Escola Paulista de Medicina.

3. Professora de Patologia, Departamento de Patologia, Universidade Federal de São Paulo, Escola Paulista de Medicina.

4. Professor Titular da Disciplina de Gastroenterologia Pediátrica, Departamento de Pediatria, Universidade Federal de São Paulo, Escola Paulista de Medicina.

Artigo submetido em 17.02.03, aceito em 28.05.03.

\begin{abstract}
Objective: to report two cases of infants with hemorrhagic gastritis due to cow's milk allergy.

Description: the clinical features included hematemesis, vomiting and malnutrition. All patients had eosinophilic infiltrate in gastric biopsies and got favorable clinical outcome after cow's milk free diet.

Comments: hemorrhagic gastritis due to cow's milk allergy is an uncommon diagnosis. Clinical findings in 10 patients (including ours) reported with allergic gastritis were vomiting, malnutrition, anemia, and hematemesis. Gastritis occurs in cow's milk allergy in a wide range of severity, and it could remain hidden in most patients. Unless appropriate management is ensued, patients develop severe malnutrition and severe anemia. Allergic compromise of upper gastrointestinal tract might be considered in all vomiting infants particularly if complicated by hematemesis. Diagnosis of allergic gastritis relies on clinical suspicion helped by endoscopy and gastric biopsies.
\end{abstract}

J Pediatr (Rio J) 2003;79(4):363-8: hemorrhagic gastritis, cow's milk allergy, infant.
As manifestações gastrointestinais de alergia alimentar incluem diarréia crônica com má absorção, sangramento retal, refluxo gastroesofágico, constipação intestinal e hemorragia digestiva alta ${ }^{1-4}$. Refluxo gastroesofágico (RGE) e alergia ao leite de vaca (ALV) são considerados os distúrbios gastrointestinais mais comuns em lactentes no primeiro ano de vida, e, muitas vezes, são patologias relacionadas ${ }^{3,5}$. Vômitos persistentes, ganho ponderal in- 
suficiente e hematêmese, em lactentes jovens, podem ser decorrentes a RGE secundário à gastrite por $\mathrm{ALV}$, manifestação pouco freqüente desta patologia ${ }^{6-10}$. Neste estudo, relatamos dois pacientes atendidos em nosso serviço, ao longo destes últimos seis anos, com gastrite hemorrágica.

\section{Descrição de casos \\ Caso 1}

T.A.S.S., sexo masculino, 24 meses, nascido a termo, sem intercorrências perinatais, com queixa de vômitos, hematêmese e perda de peso. Referia regurgitações freqüentes desde os dois meses, e, aos cinco meses, foi internado em outro hospital, devido a dois episódios de hematêmese acompanhados de palidez e irritabilidade. A partir do segundo dia de internação, as fezes tornaram-se diarréicas, sem sangue, mas apresentou melhora clínica, realizou endoscopia digestiva ao nono dia de internação, que foi relatada como exame normal; não necessitou de transfusão sanguínea e teve alta hospitalar. Manteve quadro de irritabilidade e vômitos, perdendo peso, e, após 45 dias, apresentou outro episódio de hematêmese. Não havia febre ou alterações cutâneas. Nessa ocasião, procurou nosso serviço. Exames: hemoglobina 10,2 g/dl, hematócrito 32\%, 15.600 leucócitos (neutrófilos bastonados $8 \%$, neutrófilos segmentados $47 \%$, eosinófilos 3\%, linfócitos 36\% e monócitos $6 \%$ ); os testes de coagulação apresentaram-se normais. A endoscopia digestiva alta mostrou pangastrite erosiva hemorrágica, que foi atribuída à ingestão de ácido acetilsalicílico, pois a mãe administrava regularmente $(50 \mathrm{mg} / \mathrm{dia})$ para debelar cólicas. Foi prescrito ranitidina, sulfato ferroso e recomendou-se a interrupção do uso de ácido acetilsalicílico e outros antiinflamatórios não-esteróides (AINE). Após 15 dias, retornou referindo vários episódios de vômitos com sangue. Na ocasião, o paciente apresentava escores $\mathrm{z}$ de peso para idade e estatura para idade inferiores a -2. Exame endoscópico mostrou pangastrite erosiva hemorrágica. Biópsia de antro mostrou gastrite aguda ulcerada com intenso infiltrado eosinofílico (24,1 eosinófilos /campo de grande aumento), negativo para Helicobacter pylori; biópsia de duodeno mostrou enteropatia com atrofia vilositária subtotal, e biópsia retal mostrou colite crônica inespecífica, sem infiltrado de eosinófilos. Aos 10 meses de vida, pesava $8.500 \mathrm{~g}$ e media $71 \mathrm{~cm}$ (escores $\mathrm{z}$ de peso para a idade e de estatura para a idade superiores a -1,5). A introdução de fórmula láctea ocorreu aos dois meses de idade. Não havia história familiar de alergia.

Diagnosticou-se ALV e iniciou-se dieta com exclusão da proteína do leite de vaca da dieta e introduziu-se fórmula a base de soja. Evoluiu com remissão total dos sintomas; houve recuperação nutricional e normalização dos níveis de hemoglobina. Aos dois anos de idade, foi realizada nova endoscopia e, durante o procedimento, foi infundido leite de vaca diretamente sobre a mucosa gástrica, sendo coletada biópsia gástrica neste local. Não houve alteração ma- croscópica e a histologia foi normal. Reintroduziu-se leite de vaca, permanecendo assintomático, com boa evolução ponderal e, após dois anos de seguimento, teve alta assintomático.

\section{Caso 2}

B.S.S., sexo feminino, 24 meses, encaminhada com queixa de vômitos pós-alimentares e ganho de peso insuficiente. O quadro iniciou aos dois meses de idade, com vômitos diários, com traços de sangue, duas a três vezes por semana e, em três ocasiões, referia maior quantidade de sangue. Aos três meses, diagnosticou-se RGE, recebendo tratamento com cisaprida e ranitidina. Após dez dias de tratamento, os vômitos com sangue persistiram, iniciando quadro diarréico, com comprometimento do estado geral, sendo internada em nosso serviço. Hemocultura foi positiva para Escherichia coli e iniciou-se ceftriaxone. A paciente apresentava também anemia (hemoglobina 6,2 g/l), requerendo duas transfusões sanguíneas. Pesava $5.300 \mathrm{~g}$ e media $57,5 \mathrm{~cm}$ aos cinco meses de idade (escores $\mathrm{z}$ de peso para idade e estatura para idade inferiores a -2).

A paciente nasceu a termo e apresentou icterícia neonatal, requerendo fototerapia, sem outras intercorrências. O leite de vaca integral foi introduzido no primeiro mês de vida. Não havia história familiar de atopia.

Exame endoscópico mostrou esofagite, gastrite erosiva no cárdia e na parede anterior de corpo e duodenite, sendo coletados fragmentos de esôfago, estômago e duodeno. À histologia, a mucosa esofágica mostrou congestão vascular e alguns leucócitos intra-epiteliais, a mucosa gástrica apresentava área de erosão superficial, edema, congestão vascular, infiltrado inflamatório denso e difuso, com a presença de linfócitos, plasmócitos, neutrófilos e numerosos eosinófilos (26,2 /campo de grande aumento) no epitélio glandular, no fundo das criptas e na muscularis mисоsae. Também foram observados organismos similares ao Helicobacter pylori. A mucosa duodenal exibia aumento da celularidade do córion, leve eosinofilia e áreas de discreto achatamento das vilosidades. Foi coletada também biópsia de mucosa retal, que apresentava discreto aumento do infiltrado celular do córion.

Excluíram-se derivados lácteos da dieta, houve resolução total dos sintomas e alcançou recuperação nutricional, em uso de fórmula semi-elementar. Aos dois anos de idade, foi feito desencadeamento com fórmula láctea, e a paciente permaneceu assintomática.

\section{Comentários}

Hemorragia digestiva alta é sintoma pouco freqüente em lactentes, e pode estar associada à esofagite de refluxo, gastrite por AINE, gastrite por estresse, úlcera gástrica primária e gastrite secundária à ALV. Foi descrita inicialmente por Kravis e col. ${ }^{10}$, que relatou um paciente de três meses de idade com vômitos, hematêmese e baixo ganho de 
peso, que desapareceram após a exclusão da proteína do leite de vaca da dieta. Há outros quatro relatos na literatura, envolvendo mais sete pacientes ${ }^{6-10}$ (Tabela 1).

Em todos os pacientes descritos, houve desmame precoce, nos três primeiros meses de vida, e início do quadro clínico no primeiro semestre de vida (um com hematêmese no primeiro mês). Embora alguns casos de gastrite alérgica tenham início abrupto, muitos pacientes relatados passam um período com sintomas sugestivos de refluxo, com vômitos persistentes, como observado nos dois pacientes descritos. O sintoma mais freqüente foi vômitos recorrentes, culminando, na maioria deles (7/9 pacientes), com episódios de hematêmese. Vale salientar que 22\% (2/9) dos pacientes não apresentaram hemorragia digestiva alta em momento algum de sua evolução. Portanto, lactentes com RGE refratário ao tratamento clínico podem apresentar refluxo secundário à ALV. As diretrizes da Sociedade Norteamericana de Gastroenterologia, Hepatologia e Nutrição Pediátrica incluíram dieta hipoalergênica (ensaio por uma semana) no algoritmo de manejo do lactente com $\mathrm{RGE}^{3,11}$.

Uso de antiinflamatório não-esteróide (AINE) deve ser sempre considerado na ocorrência de hemorragia digestiva alta, posto que a indicação de AINE é muito difundida no tratamento das infecções virais respiratórias em nosso meio. Tais medicações são muito tóxicas à mucosa gástrica, e estudos experimentais demonstram que dez minutos após exposição direta ao ácido acetilsalicílico, a mucosa apresenta alterações ultra-estruturais decorrentes de sua toxicidade $^{12}$. Os achados histológicos são variados, desde hiperplasia foveolar, edema, proliferação de músculo liso na camada muscular da mucosa, números normais ou levemente aumentados de células linfomononucleares e ausên-

Tabela 1 - Aspectos clínicos, histológicos e endoscópicos de oito pacientes descritos na literatura mundial e dois casos do presente relato com gastrite hemorrágica por ALV

\begin{tabular}{|c|c|c|c|c|c|}
\hline Autores & $\begin{array}{l}\mathrm{N}^{0} \mathrm{de} \\
\operatorname{casos}\end{array}$ & $\begin{array}{c}\text { Idade da } \\
\text { hematêmese (m) }\end{array}$ & $\begin{array}{l}\text { Idade do } \\
\text { desmame }\end{array}$ & $\begin{array}{c}\text { Endoscopia } \\
\text { digestiva alta (n) }\end{array}$ & Biópsias \\
\hline Kravis e col. $(1967)^{10}$ & 1 & $5 \mathrm{~m}$ & $1 \mathrm{~m}$ & Não realizado & Não realizado \\
\hline $\begin{array}{l}\text { Coello-Ramirez \& } \\
\text { Larrossa-Haro }^{6}\end{array}$ & 4 & $\begin{array}{l}1 \text { paciente } \\
(<1 \mathrm{~m})\end{array}$ & $\begin{array}{l}5 \text { a } 12 \text { dias } \\
\text { de vida }\end{array}$ & $\begin{array}{c}\text { Esofagite (1), } \\
\text { gastrite (1), } \\
\text { gastroduodenite (1), } \\
\text { erosões antrais (3) }\end{array}$ & Não realizado \\
\hline Brunerie e col. $(1986)^{7}$ & 1 & $5 \mathrm{~m}$ & $\begin{array}{c}\text { Desde o } \\
\text { nascimento }\end{array}$ & $\begin{array}{c}\text { Gastrite } \\
\text { hemorrágica } \\
\text { e bulbite } \\
\text { hemorrágica }\end{array}$ & $\begin{array}{l}\text { Antro: mucosa } \\
\text { dismórfica com } \\
\text { epitélio desdiferenciado } \\
\text { e córion inflamatório, } \\
\text { congestivo e com } \\
\text { sufusões hemorrágicas } \\
\text { intersticiais. Duodeno: } \\
\text { atrofia vilositária grau II. } \\
\text { Reto: colite }\end{array}$ \\
\hline El Mouzan e col. $(1990)^{8}$ & 1 & $3 \mathrm{~m}$ & 2 dias & Gastrite erosiva & $\begin{array}{c}\text { Antro e duodeno: } \\
\text { infiltrado inflamatório } \\
\text { com eosinofilia tecidual }\end{array}$ \\
\hline Heldenberg e col.(1993) & 1 & $5 \mathrm{~m}$ & 3 meses & $\begin{array}{l}\text { Gastrite erosiva } \\
\text { e duodenite }\end{array}$ & $\begin{array}{c}\text { Antro e duodeno: } \\
\text { infiltrado inflamatório } \\
\text { com eosinofilia tecidual }\end{array}$ \\
\hline $\begin{array}{l}\text { Machado e col. (2002) } \\
\text { Descrição atual }\end{array}$ & 2 & $2 \mathrm{~m}$ & $1-2$ meses & $\begin{array}{l}\text { Gastrite erosiva } \\
\text { ou hemorrágica } \\
\text { Duodenite (1) }\end{array}$ & $\begin{array}{l}\text { Infiltrado inflamatório } \\
\text { com eosinofilia tecidual } \\
\text { em mucosa gástrica (2), } \\
\text { atrofia vilositária (2) }\end{array}$ \\
\hline
\end{tabular}


cia de leucócitos polimorfonucleares, salvo se há erosão na área biopsiada. Por outro lado, na gastrite alérgica ocorre um aumento do infiltrado linfoplasmocitário e infiltrado neutrofílico com eosinofilia tecidual ${ }^{13}$. A resposta ao tratamento contribui para o diagnóstico diferencial, pois a hemorragia cessa logo após a suspensão do medicamento causador da gastrite medicamentosa.

Anormalidades endoscópicas, especialmente esofagite, erosões gástricas, erosões duodenais e hiperplasia nodular linfóide no duodeno são freqüentes em pacientes com alergia alimentar, e provavelmente são subdiagnosticadas ${ }^{14}$. Erosões gástricas estavam presentes em $3 / 15$ pacientes com dor abdominal e diarréia, com diagnóstico de alergia alimentar estabelecido com desencadeamento duplo-cego placebo controlado, embora nenhum deles tivesse apresentado hemorragia digestiva ${ }^{15}$. A região do antro gástrico sempre apresenta padrão inflamatório com eosinofilia tecidual, enquanto no corpo gástrico as alterações são focais ${ }^{16}$. Eosinofilia na mucosa gástrica (região gástrica biopsiada não especificada) foi descrita em $16 / 28$ pacientes por Kokkonen e col. ${ }^{14}$, contra $1 / 56$ dos controles, enquanto eosinofilia no intestino proximal foi similar nos dois grupos de pacientes. A eosinofilia da mucosa gástrica, importante ao diagnóstico, pode estar presente também em processos neoplásicos, pólipo fibróide inflamatório, doença de Crohn, poliarterite nodosa, infestações parasitárias, gastrite granulomatosa, edema hereditário angioneurótico, mastocitose sistêmica, síndrome de hipereosinofilia, histiocitose $\mathrm{X}$, e gastroenterite eosinofílica, diagnósticos pouco prováveis no primeiro semestre da vida ${ }^{17}$.

Má absorção é freqüente na ALV, e em uma casuística italiana com 73 pacientes, os sintomas mais freqüentes foram diarréia $(44 / 73,60,3 \%)$ e retardo do crescimento (24/ $73,32,9 \%)^{18}$. Nos casos descritos de gastrite alérgica (Tabela 1), diarréia (quatro pacientes) e ganho ponderal insuficiente (sete pacientes) foram sintomas comuns, indicando geralmente acometimento conjunto do intestino delgado em uma parcela dos pacientes. Mesmo nos pacientes com sintomas de gastrite iniciados de forma abrupta, foi observado retardo ponderal, sugerindo que já havia máabsorção. Todos os pacientes relatados por Coello-Ramirez e Larrosa-Haro apresentavam teste de absorção de Dxilose anormal ${ }^{6}$. Nossos dois pacientes apresentaram atrofia vilositária, um deles subtotal em biópsias de duodeno associada a infiltrado linfoplasmocitário, e alterações similares foram também descritas por Brunerie e col. ${ }^{7}$ Portanto, comprometimento do intestino delgado é freqüente e justifica o agravo nutricional encontrado entre os casos relatados.

Descrita inicialmente por Gryboski e col., a colite alérgica é uma manifestação comum de ALV, com quadro clínico típico e ocorre principalmente em lactentes jovens $^{2,10,19}$. A colite alérgica pode ocorrer com estímulo antigênico veiculado pelo leite materno, mesmo em pacientes ainda não expostos diretamente a proteínas heterólogas $^{20,21}$. O diagnóstico é auxiliado pelo exame histológico, em que há infiltrado de linfomononucleares e eosinófilos na mucosa retal ${ }^{22,23}$. Nossos pacientes não apresentavam colite alérgica, ao contrário da paciente descrita por Brunerie e col. ${ }^{7}$ Os pacientes com colite alérgica geralmente têm acometimento basicamente do intestino grosso, sem repercussão no estado nutricional, e são lactentes com aspecto saudável. Constipação intestinal pode ser uma manifestação de alergia alimentar, possivelmente pela dor evacuatória associada à inflamação do reto ${ }^{4}$.

Os mecanismos imunológicos envolvidos na enterocolite alérgica não estão bem definidos ${ }^{24}$. A alergia a antígenos presentes no alimento pode ser resultado de alterações na função linfocitária ${ }^{25}$. O leite de vaca é rico em determinantes antigênicos, sendo a beta-lactoglobulina a mais importante, a qual pode ser reconhecida pelas células $\mathrm{T}$ de pacientes alérgicos em uma variedade de epítopos ${ }^{26}$. O estímulo de células $\mathrm{T}$ por mitógenos de pacientes com alergia alimentar produz menos interferon $\gamma$ que o estímulo de linfócitos T oriundos de indivíduos normais ${ }^{27}$. Na mucosa intestinal, há também aumento na quantidade de linfócitos gama delta, assim como na relação $\gamma \delta / \mathrm{CD} 3^{15}$. A maior permeabilidade da mucosa intestinal a macromoléculas nos primeiros meses de vida atua como um precipitante em pacientes com desmame precoce. A normalização das alterações linfocitárias marca o desenvolvimento de tolerância, geralmente no fim do primeiro ano de vida ${ }^{28-30}$.

O diagnóstico de alergia alimentar classicamente é estabelecido pelo critério de Goldman, ou seja, a resolução dos sintomas sob dieta de exclusão e a recaída com o desencadeamento com o antígeno ${ }^{31,32}$. Isto pode não ser adequado em lactentes, pois o desenvolvimento de tolerância pode levar a um desencadeamento negativo, e um desencadeamento precoce pode não ser recomendado pela potencial gravidade das manifestações ${ }^{32}$. Outros exames, como a pesquisa de imunoglobulina Eespecífica, através de exames sorológicos ou de testes cutâneos, não são suficientemente sensíveis entre lactentes e não são recomenda$\operatorname{dos}^{32}$. A infusão de leite sobre a mucosa gástrica durante endoscopia pode produzir lesão macro e microscópica em curto espaço de tempo em pacientes adultos sensibilizados, mas não há estudos em crianças ${ }^{33}$.

É importante observar que a única contribuição do hemograma é a presença de anemia microcítica. Ao contrário da colite alérgica, em que até $40 \%$ dos pacientes apresentam eosinofilia importante, tal alteração não foi observada em nenhum dos pacientes relatados com gastrite alérgica $^{6-10,16}$.

A exclusão da proteína do leite de vaca produz resposta clínica importante. A dieta recomendada deve ser isenta em leite de vaca e seus derivados, e fórmulas para lactentes, compostas por hidrolisado protéico ou proteína de soja, podem ser empregadas. A maioria dos relatos na literatura refere emprego de fórmula à base de proteína da soja com sucesso, mas $40 \%$ dos pacientes podem apresentar sensibilidade cruzada ${ }^{34}$. Entre os relatos disponíveis, em apenas dois pacientes a fórmula empregada continha hidrolisado 
protéico, embora constitua a alternativa preferencialmente empregada, se disponível ${ }^{20,21}$.

A ALV compromete a mucosa gástrica em graus variáveis, desde gastrite histológica a gastrite hemorrágica, sua expressão máxima de gravidade, podendo ser subdiagnosticada. A gastrite por ALV pode estar presente em lactentes com sintomas como vômitos, irritabilidade, falha em ganhar peso, mesmo na ausência de hemorragia digestiva aparente. Odiagnóstico é eminentemente clínico, e o tratamento deve ser rapidamente instituído, em casos suspeitos. A endoscopia digestiva alta com biópsias deverá ser realizada, se a evolução não confirmar o diagnóstico.

\section{Referências bibliográficas}

1. Sampson HA. Food allergy. Part 1: immunopathogenesis and clinical disorders. J Allergy Clin Immunol 1999;103:717-28.

2. Fagundes Neto U, Martins MCV, Lima FMLS. Intolerância às proteínas heterólogas da dieta: enteropatia às proteínas do leite de vaca, soja e frango e suas manifestações clínicas. J Pediatr (Rio J) 1988;64:306-10.

3. Cavataio F, Iacono G, Montalto G, Soresi M, Tumminello M, Campagna P, et al. Gastroesophageal reflux associated with cow's milk allergy in infants: which diagnostic examinations are useful? Am J Gastroenterol 1996;91:1215-20.

4. Iacono G, Cavataio F, Montalto G, Florena A, Tumminello M, Soresi M, et al. Intolerance of cow's milk and chronic constipation in children. N Engl J Med 1998;339:1100-4.

5. Cavataio F, Carroccio A, Iacono G. Milk-induced reflux in infants less than one year of age. J Pediatr Gastroenterol Nutr 2000;30 Suppl:36-44.

6. Coello-Ramirez P, Larrosa-Haro A. Gastrointestinal occult hemorrhage and gastroduodenitis in cow's milk protein intolerance. J Pediatr Gastroenterol Nutr 1984;3:215-8.

7. Brunerie M, Olives JP, Couvaras O, Ghisolfi J. Gastroduodénite aiguë hémorragique par intolérance aux protéines du lait de vache. Arch Fr Pediatr 1986;43:291.

8. el Mouzan MI, al Quorain AA, Anim JT. Cow's-milk-induced erosive gastritis in an infant. J Pediatr Gastroenterol Nutr 1990;10:111-3.

9. Heldenberg D, Abudy Z, Keren S, Auslaender L. Cow's milkinduced hematemesis in an infant. J Pediatr Gastroenterol Nutr 1993; 17:450-2.

10. Kravis LP, Donsky G, Lecks HI. Upper and lower gastrointestinal tract bleeding induced by whole cow's milk in an atopic infant. Pediatrics 1967;40:661-5.

11. Rudolph CD, Mazur LJ, Liptak GS, Baker RD, Boyle JT, Colletti $\mathrm{RB}$, et al. Guidelines for evaluation and treatment of gastroesophageal reflux in infants and children: recommendations of the North American Society for Pediatric Gastroenterology and Nutrition. J Pediatr Gastroenterol Nutr 2001;32 Supl 2:1-31.

12. Baskin WN, Ivey KJ, Krause WJ, Jeffrey GE, Gemmell RT. Aspirin-induced ultrastructural changes in human gastric mucosa: correlation with potential difference. Ann Intern Med 1976;85: 299-303.

13. Dixon MF, Genta RM, Yardley JH, Correa P. Classification and grading of gastritis. The updated Sydney System. International Workshop on the Histopathology of Gastritis, Houston 1994. Am J Surg Pathol 1996;20:1161-81.
14. Kokkonen J, Ruuska T, Karttunen TJ, Niinimaki A. Mucosal pathology of the foregut associated with food allergy and recurrent abdominal pains in children. Acta Paediatr 2001;90:16-21.

15. Kokkonen J, Haapalahti M, Laurila K, Karttunen TJ, Maki M. Cow's milk protein-sensitive enteropathy at school age. J Pediatr 2001;139:797-803.

16. Goldman H, Proujansky R. Allergic proctitis and gastroenteritis in children. Clinical and mucosal biopsy features in 53 cases. Am J Surg Pathol 1986;10:75-86.

17. Villanacci V, Ragni F, Grigolato PG, Favret M, Marocolo D, Benetti A, et al. Gastrite eosinofila. Considerazioni clinicopatologiche su di un caso e revisione della letteratura. Minerva Med 1990;81:735-40.

18. Mancini S, Castro M, Lucidi V, Palumbo M, Dall'Oglio L. Caratteristiche cliniche in 73 bambini affetti da allergia alle proteine del lette vaccino. Minerva Pediatr 1985;37:49-52.

19. Gryboski JD, Burkle F, Hillman R. Milk induced colitis in an infant. Pediatrics 1966;38:299-302.

20. Machida HM, Catto Smith AG, Gall DG, Trevenen C, Scott RB. Allergic colitis in infancy: clinical and pathologic aspects. $\mathbf{J}$ Pediatr Gastroenterol Nutr 1994;19:22-6.

21. Odze RD, Wershil BK, Leichtner AM, Antonioli DA. Allergic colitis in infants. J Pediatr 1995;126:163-70.

22. Iyngkaran N, Yadav M, Boey CG. Rectal mucosa in cows' milk allergy. Arch Dis Child 1989;64:1256-60.

23. Berezin S, Schwarz SM, Glassman M, Davidian M, Newman LJ. Gastrointestinal milk intolerance of infancy. Am J Dis Child 1989;143:361-2.

24. Walker WA. Cow's milk protein-sensitive enteropathy at school age: a new entity or a spectrum of mucosal immune responses with age. J Pediatr 2001;139:765-6.

25. Guarderas JC. Is it food allergy? Differentiating the causes of adverse reactions to food. Postgrad Med 2001;109:125-7, 131-4.

26. Inoue R, Matsushita S, Kaneko H, Shinoda S, Sakaguchi H, Nishimura Y, et al. Identification of beta-lactoglobulin-derived peptides and class II HLA molecules recognized by T cells from patients with milk allergy. Clin Exp Allergy 2001;31:1126-34.

27. Jaffe JS, James SP, Mullins GE, Braun-Elwert L, Lubensky I, Metcalfe DD. Evidence for an abnormal profile of interleukin-4 (IL-4), IL-5, and gamma-interferon (gamma-IFN) in peripheral blood $\mathrm{T}$ cells from patients with allergic eosinophilic gastroenteritis. J Clin Immunol 1994;14:299-309.

28. Suomalainen H, Soppi E, Laine S, Isolauri E. Immunologic disturbances in cow's milk allergy, II: Evidence for defective interferon-gamma generation. Pediatr Allergy Immunol 1993;4:203-7.

29. Nagata S, Yamashiro Y, Ohtsuka Y, Shioya T, Oguchi S, Shimizu T, et al. Quantitative analysis and immunohistochemical studies on small intestinal mucosa of food-sensitive enteropathy. J Pediatr Gastroenterol Nutr 1995;20:44-8.

30. Augustin M, Karttunen TJ, Kokkonen J. TIA1 and mast cell tryptase in food allergy of children: increase of intraepithelial lymphocytes expressing TIA1 associates with allergy. J Pediatr Gastroenterol Nutr 2001;32:11-8.

31. Goldman AS, Anderson Jr. DW, Sellers WA, Saperstein S, Knicker WT, Halpern SR. Milk allergy I. Oral challenge with milk and isolated milk proteins in allergic children. Pediatrics 1963;32:425-43.

32. Diagnostic criteria for food allergy with predominantly intestinal symptoms. The European Society for Paediatric Gastroenterology and Nutrition Working Group for the Diagnostic Criteria for Food Allergy. J Pediatr Gastroenterol Nutr 1992;14:108-12. 
33. Reimann HJ, Lewin J. Gastric mucosal reactions in patients with food allergy. Am J Gastroenterol 1988;83:1212-9.

34. Halpern SR, Sellars WA, Johnson RB, Anderson DW, Saperstein S, Reisch JS. Development of childhood allergy in infants fed breast, soy, or cow milk. J Allergy Clin Immunol 1973;51:139-51.
Endereço para correspondência:

Dr. Rodrigo Strehl Machado

Rua Loefgreen, 1596

CEP 04040-032 - São Paulo, SP

Tel.: (11) 5573.3757 - Fax: (11) 5573.8998

E-mail: rodrigo@gastroped.epm.br 